Research Article

\title{
Undernutrition and Associated Factors among Lactating Mothers in Dega Damot Woreda, Northwest Ethiopia
}

\author{
Hunegnaw Almaw Derseh $\mathbb{D}^{1},{ }^{1}$ Liknaw Bewket Zeleke $\mathbb{D}^{2},{ }^{2}$ Melese Linger Endalifer $\left(\mathbb{D},{ }^{3}\right.$ \\ Esmael Ali Muhammad $\left(\mathbb{D},{ }^{4}\right.$ and Terefe Derso ${ }^{4}$ \\ ${ }^{1}$ College of Health Sciences and Medicine, Bahir Dar University, Bahir Dar, Ethiopia \\ ${ }^{2}$ College of Health Sciences, Debre Markos University, Debre Markos, Ethiopia \\ ${ }^{3}$ Department of Public Health, Faculty of Health Science, Woldia University, Woldia, Ethiopia \\ ${ }^{4}$ College of Health Sciences and Medicine, University of Gondar, Gondar, Ethiopia
}

Correspondence should be addressed to Hunegnaw Almaw Derseh; hunsew25@gmail.com

Received 12 June 2020; Revised 26 August 2020; Accepted 23 October 2020; Published 6 November 2020

Academic Editor: C. S. Johnston

Copyright ( $) 2020$ Hunegnaw Almaw Derseh et al. This is an open access article distributed under the Creative Commons Attribution License, which permits unrestricted use, distribution, and reproduction in any medium, provided the original work is properly cited.

\begin{abstract}
Introduction. Nutritional status of lactating women is highly linked with the overall nutritional status of population. Undernutrition during lactation results in increased morbidity and mortality to the mother and her child. The literature is dearth regarding to nutrition status of lactating mothers in developing countries such as Ethiopia. Objective. The aim of this study was to assess undernutrition and associated factors among lactating mothers having child 6-23 months in Dega Damot Woreda, Northwest Ethiopia. Methods. A community-based cross-sectional study was conducted from March 21 to April 19, 2018. Simple random sampling technique was employed to recruit a total of 733 participants. Data were collected by using an interviewadministered questionnaire and entered into EpiData version 4.2 and transferred to SPSS version 20 for further analysis. Factors associated with undernutrition were identified using multivariable logistic regression at a $p$ value of less than 0.05 at $95 \%$ confidence interval. The direction and strength of association was interpreted using adjusted odds ratio. Results. In this study, the magnitude of undernutrition among lactating women was $21.8 \%$. Being unmarried (AOR: 2.65, 95\% CI: 1.54, 4.53), their first pregnancy $<18$ years (AOR: 2.34, 95\% CI: 1.42, 3.87), having more than 5 number of pregnancies (AOR: 2.79, 95\% CI: 1.55, 5.03), inadequate dietary diversity score (AOR: $2.1,95 \%$ CI: 1.16, 3.83), and household food insecurity (AOR: 3.68, 95\% CI: 2.35, 5.77) were significantly associated factors with undernutrition. Conclusions. The prevalence of undernutrition among lactating women was found to be high. Programs should do better in enhancing activities of agricultural production to reduce household food insecure and to increase accessibility of diversified diet. Other researchers better to do other studies to identify main factors of undernutrition using a qualitative study.
\end{abstract}

\section{Introduction}

Undernutrition is a clinical syndrome characterized by weight loss associated with significant depletion of fat stores and muscle mass [1]. Worldwide, child and maternal undernutrition remain unacceptably high. An undernourished woman up to the second birth day of the child is a threat to woman's health, child survival, growth, development, and productivity in later life [2]. Globally in every year, an estimated 14 million adolescent girls enter to lactation from which more than $90 \%$ live in developing countries [3].
Malnutrition among women is likely to have a major impact on their own health as well as their children's health. More than 3.5 million women and children under age five in developing countries die each year due to the underlying cause of undernutrition [4]. Undernourished women give a sick and cognitively deficient child and responsible for 800,000 neonatal deaths through small for gestational age births [5]. Women in developing countries with underweight have a high risk of mortality and morbidity, and it also affects the quality and volume of breast milk produced; undernourished woman produce less amount of breast milk 
than the well-nourished woman [6]. Evidences indicated that women with a body mass index $(\mathrm{BMI}) \leq 18.5 \mathrm{~kg} / \mathrm{m}^{2}$ are at increased risk of illness and mortality rate [7]. Health and nutritional status of women highly linked with the overall health and nutritional status of population [8].

Since women in middle- and low-income countries are nutritionally vulnerable groups for the increased nutritional demand of lactation, developing countries including Ethiopia give an especial focus to appropriate nutrition during lactation [9].

Women of reproductive age are especially vulnerable to chronic energy deficiency and malnutrition that leads to low productivity among adults, and also, it is a major risk factor for adverse birth outcomes [10]. According to WHO cutoff values (20-39\%) for public-health significance of women underweight, Ethiopia has high prevalence or serious situation of women undernourishment and the country has the second highest rate of malnutrition in Sub-Saharan Africa $[3,6,11]$.

The literature is dearth regarding to nutrition status of lactating mothers in developing countries such as Ethiopia; as a result, this study was conducted to investigate the magnitude of undernutrition and its associated factors among lactating mothers in Dega Damot Woreda, Northwest Ethiopia, which might provide some information regarding to this issue.

\section{Methods}

2.1. Study Area. This study was conducted in Dega Damot Woreda, Northwest Ethiopia. The administrative center of the woreda is Feres Bet Town which is located around $250 \mathrm{~km}$ from Bahir Dar, the capital city of Amhara Region and $399 \mathrm{~km}$ from Addis Ababa, the capital city of Ethiopia. The woreda has 32 rural and 2 urban kebeles. Based on the 2010 E.C. woreda health office quarter population report, from the total population of the woreda, lactating mothers having children 6-23 months old were accounting 4.4\% [12].

2.2. Study Design and Period. A community-based crosssectional study was conducted from March 21 to April 19, 2018.

2.3. Population. The study populations of the study were all lactating mothers whose children were 6-23 months of age in Dega Damot Woreda, Amhara Region.

2.4. Inclusion and Exclusion Criteria. All lactating mothers who have children aged 6-23 months and lived for a minimum of six months were included, and lactating mothers with confirmed pregnancy were excluded.

2.5. Sample Methods and Procedure. Sample size for the first specific objective of the study was calculated using single population proportion formula determined by using the prevalence of underweight $25.4 \%(p=0.254)$ [13] with $4 \%$ marginal error $(d=0.004)$ and $95 \%$ CI using the formula
$\mathbf{n}=\left((\mathbf{Z} \cdot(\mathbf{a} / \mathbf{2}))^{\mathbf{2}} \mathbf{p} \cdot(\mathbf{1}-\mathbf{q})\right) / \mathbf{d}^{2}$. By adding $10 \%$ nonresponse rate and taking design effect of 1.5 , the calculated sample size for the first objective was 752. The sample size was also calculated for the second specific objective through considering different factors which were significantly associated with the outcome variable with $95 \%$ level of confidence, ratio of unexposed to exposed $1: 1$, and power of $80 \%$, and taking in account $10 \%$ nonresponse rate and design effect of 1.5 , the calculated sample size for the second specific objective was found to be 542. Lastly, by taking the larger from the two calculated simple size, the final sample size of this study was 752.

Multistage sampling technique was employed. First, the kebeles (the least local administrative structure) were stratified into rural and urban. Then, nine kebeles among 34 kebeles of the woreda were selected randomly by using a lottery method. The list of lactating women having children aged 6-23 months old in the selected kebele was identified from health extension worker's family folder, and the study participants were allocated to each selected kebele by using population proportion to size of each kebele. By using households which contain at least one lactating mother as sampling frame and considering lactating mother in the household as sampling unit, simple random sampling technique was employed to select study participants.

2.6. Data Collection Tools and Methods. The data were collected using a structured interview-administered Amharic questionnaire which was initially prepared in English language; after review of different related literatures' and modified based on local situations. The data collection tool prepared in English was translated to Amharic language and again back to English to check its consistency. Data on sociodemographic and economic variables, and WASHrelated characteristics were collected by using a structured questionnaire.

Level of household food insecurity was measured using the Household Food Insecurity Access Scale (HFIAS), a structured, standardized, and validated tool developed by FANTA III [14]. Dietary diversity was measured by qualitative recall of all food types consumed a day (24 hours) before the data collection date. Ten food groups were used to measure dietary diversity score which is developed by FAO and FANTA III. [15]. Attention was given to exclude special days of food consumption such as ceremonial days. Women's nutrition education was assessed by asking whether they had got or not any type of nutrition education.

Household wealth index was performed to assess socioeconomic status of respondents by asking them about the household assets, land availability and production rate, utilities, and housing characteristics.

Anthropometric measurement was carried out to determine the nutritional status of the study participants by using the body mass index (BMI). Measurement was taken at the end of the interview, and data were collected by using standardized techniques and calibrated equipment to measure weight and height of lactating women. The weight of mothers was measured to the nearest $100 \mathrm{~g}$ on a calibrated 
portable digital scale (the UNICEF electronic weighing scale) after removing shoes and wearing light closes. The height of the study participant was measured to the nearest $0.1 \mathrm{~cm}$ in erect position with their shoulder level, hands at the side, head, scapula, buttock, and heel was in contact with vertical measuring board with sliding head bar.

The data were collected through a face-to-face interview by 9 data collectors who have diploma in nursing and living in the woreda under the supervision of two BSc holder health officers after receiving training for two days by principal investigators in Amharic language about methods of anthropometric measurement, interviewing technique and filling questionnaires, and how to approach respondents.

2.7. Data Quality Control. Data quality was assured initially through careful design of the questionnaire and data collection procedure. Data collectors and supervisors were given two-day training regarding to instruments, ways of data collection and anthropometric measurement, ethical issues, and aims of the study by the principal investigator. A pretest was done prior to the actual data collection period among $5 \%(n=38)$ of the study sample. Every height and weight measurement of lactating mother was conducted twice, and the average of the measurements was taken. Proper function of digital weight scales was checked every time before the start of weight measurement, and ensuring the reading scale is exactly being at zero, checking by using a portable standard weight of $1 \mathrm{~kg}$. Supervision was carried out by two BSc health officers, and they were continuously checking completeness, accuracy, and consistency of data throughout the data collection period. Overall, the principal investigator was available to respond and clarify any concern from the data collectors and supervisors.

2.8. Data Analysis. Data entry was made using EpiData version 4.2 software after checking for completeness and consistency and then exported to SPSS version 20 software for analysis. Principal component analysis was used to determine the wealth status of respondents, and eigenvalues were used to compute wealth status of respondents for the components. Descriptive analysis was done using numerical summary measures and presented using frequency tables and figures. The body mass index of the mother was calculated through weight in kilogram divided by square of height in meters, and based on the result, the mother was categorized into undernutrition with $\mathrm{BMI}<18.5 \mathrm{~kg} / \mathrm{m}^{2}$ [16]. Bivariable logistic regression analysis was used to determine the association of independent variables with the dependent variable. Variables with $p<0.2$ in the bivariable analysis were entered into multivariable analysis to identify independent predictors of under nutrition. Lastly, variables showed $p<0.05$ in multivariable analysis were considered as significantly associated with undernutrition. Model fitness was checked using the Hosmer-Lemshow test $(p=0.117)$.

2.9. Ethical Consideration. The proposal of the study was reviewed and approved by the University of Gondar College of Health and Medical Sciences research ethical review committee to obtain ethical clearance. The ethical clearance was submitted to the Amhara region institutional health research ethical review committee to receive official letter, and the official letter was submitted to the Dega Damot Woreda health office. Data collection was started after the cooperation letter was obtained from the Woreda heath office to contact heath extension workers and participants of selected the kebele. Informed, voluntary, and written consent was gotten from lactating mothers who were aged $>18$ years, and for those lactating mothers whose ages were $<18$ years, consent was gotten from her husband/guardian after explaining the aim, duration, and any possible risks of the study through local language, Amharic. Volunteers were interviewed, and finally, personal identifiers were kept anonymously to maintain confidentiality.

\section{Result}

3.1. Sociodemographic Characteristics. A total of 733 lactating women having children aged 6 to 23 months old were involved in this study making a response rate of $97.5 \%$. The median age of respondents was 28.00 years, and more than half $(56.9 \%)$ of respondents were in the age group of 20 to 29 years. Majority of the respondents $(88.8 \%)$ were married, and about 329 (44.9\%) were unable to read and write. More than half of the respondents (55.8\%) were farmers, and 244 (33.3\%) of the respondents were poor in the wealth status (Table 1).

3.2. Obstetric Characteristics. The mean age of respondent at the first marriage was 17.54 ( $\mathrm{SD} \pm 3.11$ years). About 333 (45.4\%) of respondents were married before their eighteenth years. The median age at first pregnancy was 20 years. Two thirds $(66 \%)$ of respondents attended antenatal care for the current child. More than a half $(54.6 \%)$ of respondents delivered at home. Around three-fifth of the respondents (61\%) were using any type of family planning method during the time of data collection (Table 2).

\subsection{Water, Sanitation, and Hygienic Practice of Respondents.} Regarding to hygienic practice of respondents, about 699 (95.4\%) of respondents had latrine; off which, less than onethird (29.4\%) had hand washing facility and only 93 (13.3\%) were improved type of latrine facility. More than half, 419 (57.2\%), of respondents were practiced hand washing after toilet, and $44.7 \%$ of respondents were using unimproved source of water for drinking and cooking. Around threefourth $(74.2 \%)$ of respondents washed their food (fruits and vegetables) before they have cooked or eaten, and most of respondents (91.4\%) washed materials that they used for food preparation (Figure 1).

3.4. Health Information and Feeding Practice of Respondents. Regarding household food source, 513 (70\%) were sourced from own production. Nearly three-fourth of respondents (73.2\%) received nutritional education. Nearly one-fourth (23.5) of respondents ate additional foods during lactating. 
TABLE 1: Sociodemographic characteristics of the respondents among lactating women had children aged 6 to 23 months in Dega Damot Woreda, Northwest Ethiopia, March-April $2018(n=733)$.

\begin{tabular}{|c|c|c|}
\hline Characteristics & Frequency & Percent \\
\hline \multicolumn{3}{|l|}{ Maternal age } \\
\hline Age 15 to 19 & 11 & 1.5 \\
\hline Age 20 to 29 & 417 & 56.9 \\
\hline Age 30 to 39 & 269 & 36.7 \\
\hline Age 40 to 49 & 36 & 4.9 \\
\hline \multicolumn{3}{|l|}{ Residence } \\
\hline Urban & 197 & 26.9 \\
\hline Rural & 536 & 73.1 \\
\hline \multicolumn{3}{|l|}{ Marital status } \\
\hline Married & 651 & 88.8 \\
\hline Single & 12 & 1.6 \\
\hline Divorced & 68 & 9.3 \\
\hline Widowed & 2 & .3 \\
\hline \multicolumn{3}{|l|}{ Maternal educational status } \\
\hline Unable to read and write & 329 & 44.9 \\
\hline Read and write & 150 & 20.5 \\
\hline Primary school & 114 & 15.6 \\
\hline Secondary school & 87 & 11.9 \\
\hline College and university & 53 & 7.2 \\
\hline \multicolumn{3}{|l|}{ Husband educational status } \\
\hline Unable to read and write & 228 & 35.1 \\
\hline Able to read and write & 183 & 28.2 \\
\hline Primary school & 112 & 17.3 \\
\hline Secondary school & 51 & 7.9 \\
\hline College and university & 75 & 11.6 \\
\hline \multicolumn{3}{|l|}{ Maternal occupation } \\
\hline Farmer & 404 & 55.8 \\
\hline Housewife & 189 & 25.8 \\
\hline Merchant & 68 & 9.3 \\
\hline Government employee & 60 & 8.2 \\
\hline Self-employed & 3 & 0.4 \\
\hline Unemployed & 4 & 0.5 \\
\hline \multicolumn{3}{|l|}{ Family size } \\
\hline$<5$ & 366 & 49.9 \\
\hline$\geq 5$ & 367 & 50.1 \\
\hline \multicolumn{3}{|l|}{ Wealth index } \\
\hline Poor & 244 & 33.3 \\
\hline Medium & 245 & 33.4 \\
\hline Rich & 244 & 33.3 \\
\hline
\end{tabular}

Nearly three-fourth of respondents (77.1\%) had inadequate dietary diversity score, and $196(26.7 \%)$ took <3 time meal per day (Table 3).

3.5. Nutritional Status of Study Participants. The median BMI was $20.66 \mathrm{~kg} / \mathrm{m}^{2}$, and nearly one out of five respondents $(21.8 \%)$ had $\mathrm{BMI}<18.5 \mathrm{~kg} / \mathrm{m}^{2}$. Hence, the prevalence of undernutrition among lactating mothers whose children were aged 6-24 months in Dega Damot Woreda was 21.8\% (Figure 2).

3.6. Factors Associated with Undernutrition. The result of bivariable analysis showed that there was significant association between maternal age, marital status, age of first marriage, age of first pregnancy, number of pregnancy,
TABLE 2: Obstetric factors of the study respondents among lactating women had children aged 6 to 23 months in Dega Damot Woreda, Northwest Ethiopia, March-April $2018(n=733)$.

\begin{tabular}{|c|c|c|}
\hline Variables & Frequency & Percent \\
\hline \multicolumn{3}{|c|}{ Age at first marriage } \\
\hline$<18$ years & 333 & 45.4 \\
\hline$\geq 18$ years & 400 & 54.6 \\
\hline \multicolumn{3}{|c|}{ Age of first pregnancy } \\
\hline$<18$ years & 144 & 19.6 \\
\hline$\geq 18$ Years & 589 & 80.4 \\
\hline \multicolumn{3}{|c|}{ Number of pregnancy } \\
\hline$\leq 5$ & 648 & 88.4 \\
\hline$>5$ & 85 & 11.6 \\
\hline \multicolumn{3}{|c|}{ Attend ANC for the last pregnancy } \\
\hline Yes & 484 & 66.0 \\
\hline No & 249 & 34.0 \\
\hline \multicolumn{3}{|c|}{ Number of ANC visit $(n=478)$} \\
\hline$\leq 3$ times & 261 & 54.6 \\
\hline$>3$ times & 217 & 45.4 \\
\hline \multicolumn{3}{|c|}{ Place of delivery of current child } \\
\hline Home & 400 & 54.8 \\
\hline Health post & 18 & 2.5 \\
\hline Health center & 231 & 31.6 \\
\hline Hospital & 81 & 11.1 \\
\hline \multicolumn{3}{|c|}{ Attend PNC for the current child } \\
\hline Yes & 378 & 51.9 \\
\hline No & 350 & 48.1 \\
\hline \multicolumn{3}{|c|}{ Current FP utilization status } \\
\hline Yes & 447 & 61.0 \\
\hline No & 286 & 39.0 \\
\hline \multicolumn{3}{|c|}{ Type of FP service used } \\
\hline Pills & 46 & 10.3 \\
\hline Injectable & 376 & 84.5 \\
\hline Implant & 16 & 3.6 \\
\hline Breast feeding & 7 & 1.6 \\
\hline \multicolumn{3}{|c|}{ Number of breastfeeding } \\
\hline$<8$ times/day & 353 & 48.2 \\
\hline$\geq 8$ times/day & 380 & 51.8 \\
\hline
\end{tabular}

antenatal care for the last pregnancy, nutritional education, place of delivery, family size, hygienic practice, dietary diversity score, and food security status $(p<0.2)$. However, after controlling for possible confounders, the result of multivariable logistic regression analysis revealed that marital status, age at first pregnancy, number of pregnancy, dietary diversity score, and food security status were significantly associated with undernutrition $(p<0.05)$ (Table 4).

Lactating mothers who were unmarried were 2.65 times (AOR: 2.65, 95\% CI: 1.54, 4.53) more likely to become undernourished than those lactating women who were married. Lactating women who encountered their first pregnancy before the age of 18 years were 2.34 times (AOR: $2.34,95 \%$ CI: $1.42,3.87)$ more likely to become undernourished than those lactating women who encountered their first pregnancy at age of 18 years and above. Lactating women who had greater than 5 number of pregnancies were 2.79 times (AOR: 2.79, 95\% CI: 1.55, 5.03) more likely to be undernourished as compared to those women who had 5 


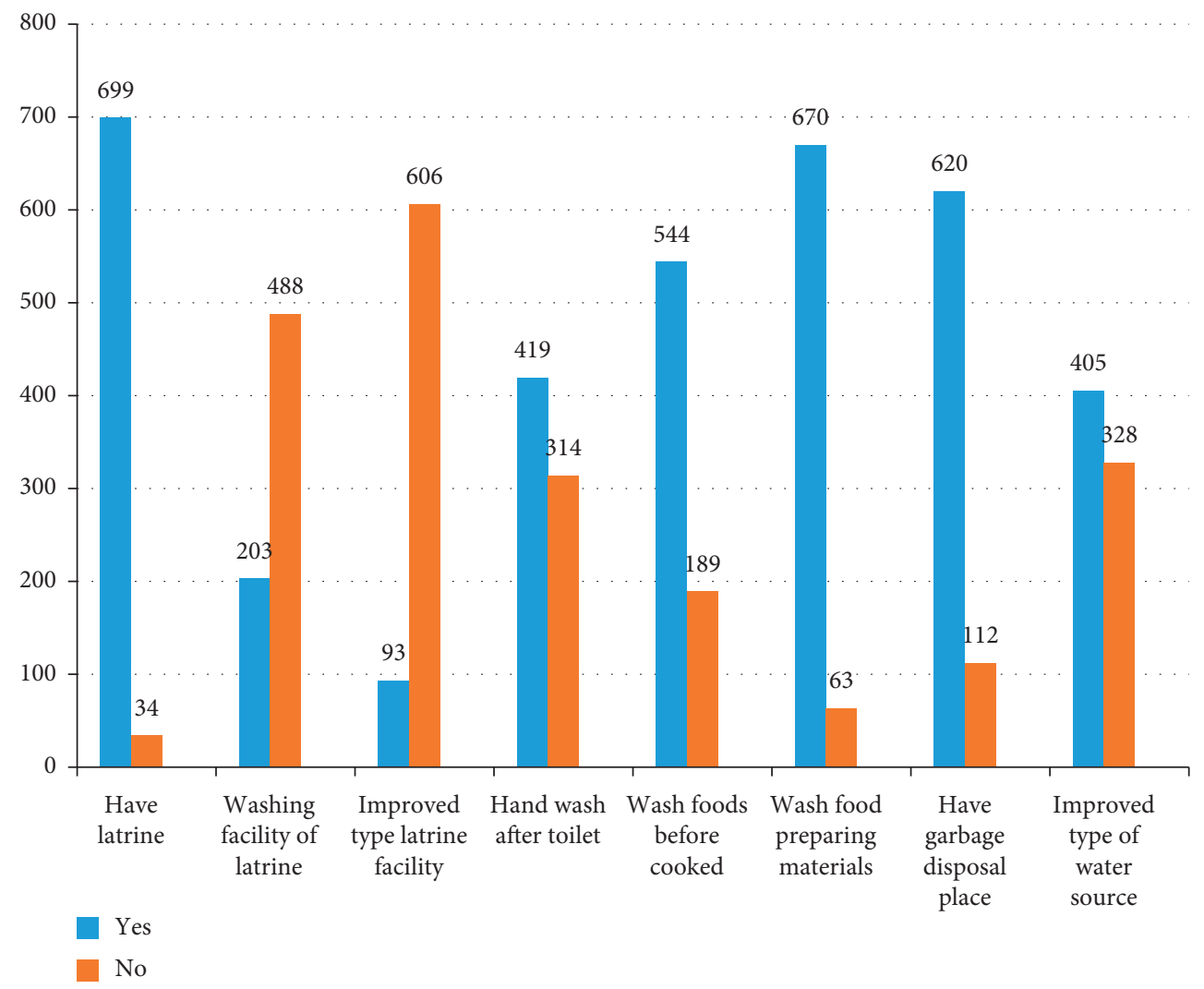

Figure 1: Water, sanitation, and hygienic practice of study participants among lactating women in Dega Damot Woreda, Northwest Ethiopia, March-April $2018(n=733)$.

TABLE 3: Health information and feeding practice of study participants among lactating women who had children aged 6 to 23 months in Dega Damot Woreda, Northwest, Ethiopia, March-April $2018(n=733)$.

\begin{tabular}{lcc}
\hline Variables & Frequency & Percent \\
\hline $\begin{array}{l}\text { Source of food for household } \\
\text { Own production }\end{array}$ & 513 & 70 \\
Purchasing & 220 & 30 \\
\hline Nutrition education & & \\
Yes & 535 & 73.2 \\
No & 196 & 26.8 \\
\hline Source of nutrition education & & \\
Health professional & 503 & 95.3 \\
Media & 9 & 1.7 \\
Peer & 16 & 3.0 \\
\hline Eat any additional food during lactating & \\
Yes & 172 & 23.5 \\
No & 559 & 76.5 \\
\hline Avoid eating any food during lactating & \\
Yes & 8 & 1.1 \\
No & 724 & 98.9 \\
\hline Dietary diversity score & & \\
Adequate & 168 & 22.9 \\
Inadequate & 565 & 77.1 \\
\hline Number of meals/day & & \\
$<3$ meals & 196 & \\
$\geq 3$ meals & 537 & \\
\hline
\end{tabular}

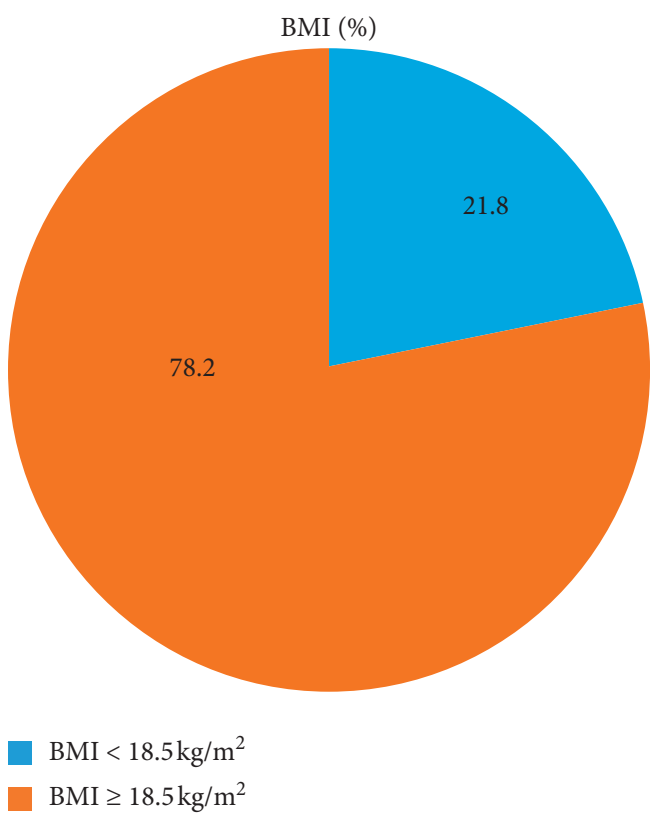

FIgURE 2: Nutritional status of lactating women in Dega Damot Woreda, Northwest Ethiopia, March-April $2018(n=733)$.

and less pregnancies. Lactating women with inadequate dietary diversity score were 2 times (AOR: 2.1, 95\% CI: 1.16, 3.83) more likely to experience undernutrition than those 
TABLE 4: Multivariable logistic regression analysis showing factors associated with undernutrition among lactating women who had children aged 6 to 23 months in Dega Damot Woreda, Northwest Ethiopia, March-April 2018 ( $n=733)$.

\begin{tabular}{|c|c|c|c|c|}
\hline \multirow{2}{*}{ Variables } & \multicolumn{2}{|c|}{ Undernutrition } & \multirow{2}{*}{ COR $(95 \% \mathrm{CI})$} & \multirow{2}{*}{$\operatorname{AOR}(95 \% \mathrm{CI})$} \\
\hline & Yes & No & & \\
\hline \multicolumn{5}{|l|}{ Marital status } \\
\hline Unmarried & 31 & 51 & $2.46(1.51,4.00)$ & $2.65(1.54,4.53)^{*}$ \\
\hline Married & 129 & 522 & 1 & 1 \\
\hline \multicolumn{5}{|c|}{ Age of first pregnancy } \\
\hline$<18$ years & 61 & 83 & $3.64(2.45,5.40)$ & $2.34(1.42,3.87)^{*}$ \\
\hline$\geq 18$ years & 99 & 490 & 1 & 1 \\
\hline \multicolumn{5}{|l|}{ Have ANC visit } \\
\hline No & 72 & 177 & $1.83(1.28,2.62)$ & $1.55(0.93,2.56)$ \\
\hline Yes & 88 & 396 & 1 & 1 \\
\hline \multicolumn{5}{|c|}{ Number of pregnancy } \\
\hline$>5$ pregnancies & 43 & 42 & $4.65(2.90,7.43)$ & $2.79(1.55,5.03)^{*}$ \\
\hline$\leq 5$ pregnancies & 117 & 531 & 1 & 1 \\
\hline \multicolumn{5}{|l|}{ Place of delivery } \\
\hline Home & 96 & 307 & $1.30(0.91,1.85)$ & $0.63(0.38,1.05)$ \\
\hline Health facility & 64 & 266 & 1 & 1 \\
\hline \multicolumn{5}{|l|}{ Dietary diversity } \\
\hline Inadequate & 145 & 420 & $3.52(2.00,6.18)$ & $2.10(1.16,3.83)^{*}$ \\
\hline Adequate & 15 & 153 & 1 & 1 \\
\hline \multicolumn{5}{|c|}{ Food security status } \\
\hline Insecure & 63 & 61 & $5.45(3.60,8.24)$ & $3.68(2.35,5.77)^{*}$ \\
\hline Secure & 97 & 512 & 1 & 1 \\
\hline
\end{tabular}

*Significant at $p$ value $<0.05$; 1 : reference group; AOR: adjusted odds ratio; COR: crude odds ratio.

women with adequate dietary diversity score, and lactating women with food insecure were 3.68 times (AOR: 3.68, 95\% CI: $2.35,5.77)$ more likely to become undernourished as compared to those food-secured women (Table 4).

\section{Discussion}

Malnutrition among women is likely to have a major impact on their own health as well as their children's health, and more than 3.5 million women and children under age five in developing countries die each year due to the underlying cause of undernutrition [17]. Women in middle- and lowincome countries are nutritionally vulnerable groups because of increase in nutritional demand of lactation [9]. In Ethiopia, some studies showed that the prevalence of underweight among lactating women ranges from 2040.6\%. According to WHO cutoff values (20-39\%) for public-health significance of women underweight, Ethiopia has high prevalence or serious situation of women undernourishment $[3,6]$.

This study was conducted to assess prevalence and associated factors of undernutrition among lactating women having children aged 6 to 23 months in Dega Damot Woreda, Northwest Ethiopia. Accordingly, the prevalence of undernutrition among lactating women was 21.8\% (95\% CI: $18.7 \%, 24.8 \%$ ) and being unmarried, early pregnancy, multigravida, inadequate dietary diversity score, and household food insecurity were significantly associated factors with undernutrition.

Nutritional demands of lactating women are greater than any stages of women's reproductive life because lactating woman produce 700 to $800 \mathrm{ml}$ of milk/day, and this requires extra energy need of about 500 calories/day [18]. Despite the higher nutritional requirements, large numbers of lactating mothers, particularly in Sub-Saharan Africa, are extremely vulnerable to nutritional deficiencies. Ethiopia is one of the developing countries of Sub-Sahara Africa having the highest level of lactating mother undernutrition; one out of four lactating women is undernourished [19]. This is also true in the present study; in which, the prevalence of undernutrition among lactating women was $21.8 \%$. This prevalence is consistent with the overall national prevalence (22\%) and prevalence of Amhara Region (22.9\%) [10], and findings from Oromia (19.5 and 20.5\%) [3,11] and Tigray Region (24.6\%) [20].

However, the prevalence of this study finding is lower from a study conducted in Womberma Woreda $(25.4 \%)$, Amhara Region [13], and Samre Woreda, Tigray (31\%) [21], and in Oromia Region, Dedo and Seqa-Chekorsa districts (40.6\%) [6], and in India (40-58\%) [22]. The reason for this difference could be educational status difference of respondents in which the respondents with no education (unable to read and write) in these studies were higher than the current study. The other possible reason for the difference may be health information (nutrition education) of respondents; in the current study, participants who got nutrition education were high. The difference also might be in occupation, wealth status, food security status, health care practice such as family planning utilization, postnatal care visit, place of delivery, and toilet utilization characteristic difference between the current study and these studies. Culture 
could also be a reason for the difference especially as compared to Indians.

On the other way, the prevalence of this study finding is higher than findings from Raya District (17.5\%) [20], research studies conducted in different African countries, Tanzania (11\%) [8], Nigeria (1.25\%) [16], and Burkina Faso (11.1-17.1\%) [23], and research conducted in Tibet (10.3\%) [24]. The probable reason for this difference could be difference in study design in which study subjects who were selected from institution might get nutrition information about nutritional requirements during lactation and importance of eating diversified food items; thus, they might be less likely to become undernourished. Also, difference in educational status of study subjects (no education in Tanzania 19\% Vs. $44.9 \%$ current study), occupation (farmer in Nigeria $4.6 \%$ Vs $55.8 \%$ current study), sample size (240 Nigeria Vs. current study 733), health care practice difference between study subjects. Another possible reason could be that the food security status, dietary diversity score, and intake of additional food during lactation of respondents in other studies were better [23].

Food insecurity is a critical variable for understanding the nutritional status of low-income populations, and it is an important determinant of undernutrition $[25,26]$. In this study also, analysis of factors associated with undernutrition among lactating women revealed that respondents with household food insecure were 3.68 times more likely to be undernourished as compared to those households with food secured. This finding is supported by a research conducted in Kunama population, Ethiopia [25], and Ghana [27]. This might be because households with food insecure are not able to access sufficient food for an active and healthy life, and limited availability of food results in inadequate dietary intake which is the immediate cause of undernutrition.

Respondents with greater than five numbers of pregnancies were 2.8 times more likely to be undernourished than those who had five and less than five. This finding is similar to another study conducted in Ethiopia, [4].This might be because of nutritional depletion of the mother due to successive pregnancies. As the mother becomes pregnant, there is a loss of nutrients, and as the number of pregnancy increases, the loss of nutrients also increases, thus leads to undernutrition.

Marital status of the women is associated with household headship and other social and economic status of the women that affects their nutritional status [28]. The result of this study showed that unmarried women were 2.7 times more likely to be undernourished as compared to those married women. This finding is supported by a study conducted in Womberma, Ethiopia [13], and the Ethiopian demographic health survey. This could be because of being married profoundly enhances family income and wealth [29], and unmarried women are poor in economic status and prone to heavy workloads both at inside and outside of the house during the lactating period which hinders to take adequate amount of diet and less number of meals in a day that the lactating women normally recommended to take.

Nutritional status at the time of conception is a culmination of a girl's life time nutritional experience, and teenage girls have the least favorable diets of all age groups [30]. The finding of this study is not surprised that women who got pregnant before the of age 18 years were 2.3 times more likely to be undernourished as compared to those women who got pregnant at the age of 18 and above. This could be because of women with young age have no welldeveloped anatomical and physiological body conditions to support pregnancy which may in turn leads to complications during pregnancy and delivery. As complications occurred, there is influence of overall health status of the lactating women and loss of nutrients which finally leads to undernourished [13]. Another reason for this might be nutrient competition between the mother and the child resulted from increased nutrient requirement for fast growth during adolescence.

Dietary monotony (less diverse diet) is an important determinant of undernutrition, and in Ethiopia, under nutrition is a main public-health catastrophe due to most people are addicted to nondiversified diet $[25,31]$. The result of this study also showed that lactating women with inadequate dietary diversity score were 2.1 times more likely to be undernourished than those who had adequate dietary diversity score. This study is supported by UNICEF's 2016 conceptual framework of malnutrition; the immediate causes of maternal malnutrition are inadequate dietary intake and disease [32]. This finding is also supported by studies in Tigray, Ethiopia, shown inadequate dietary intake among lactating mothers were more likely to be undernourished $[14,33]$.This could be because of women with inadequate dietary intake cannot fulfill the nutritional requirements for the breastfeeding children and the cells to perform its proper function.

As a limitation, the study described undernutrition by BMI measurement; thus, we recommend other researchers to examine undernutrition using other measurement techniques such as biochemical (laboratory) methods that can better detect nutritional depletions.

\section{Conclusions}

The prevalence of undernutrition among lactating women was found to be high. Being unmarried, first pregnancy before 18 years old, greater than five pregnancies, inadequate dietary diversity, and household food insecure were risk factors associated with undernutrition. Programs should do better on enhancing activities on crop production to reduce household food insecure status and to increase dietary diversity score, awareness creation about the effect of teenage pregnancy, multigravida, and importance of family planning service utilization to tackle maternal undernutrition. The other researchers better to do large-scale community-based studies for a better understanding of the nutritional status of lactating women and main factors of undernutrition using qualitative study.

\section{Abbreviations}

BMI: $\quad$ Body mass index

WHO: World Health Organization 
HFIAS: Household Food Insecurity Access Scale FANTA: Food and Nutrition Technical Assistance UNICEF: United Nations International Children's Emergency Fund

\section{Data Availability}

The data used to support the findings of this study are available from the corresponding author upon request.

\section{Ethical Approval}

The University of Gondar research ethics review committee reviewed the ethical acceptability of the research, and informed consent was obtained from each participant during data collection.

\section{Conflicts of Interest}

The authors declare that they have no conflicts of interest.

\section{Authors' Contributions}

Hunegnaw Almaw Derseh wrote the proposal, participated in data collection, analyzed the data, and drafted the paper and the manuscript. Terefe Derso and Esmael Ali Muhammad approved the proposal with some revisions, participated in data analysis, and revised subsequent drafts of the paper and manuscript. Liknaw Bewket Zeleke participated in data collection, data analysis, and drafting the paper and the manuscript. All authors read and approved the final manuscript. Melese Linger Endalifer participated in data analysis and drafting the paper and the manuscript.

\section{Acknowledgments}

The authors are very grateful to the University of Gondar for the financial support to this study and all study participants for their commitment in responding to our questionnaire.. The university supported the study by ensuring the ethical acceptability and financially covered the costs for data collector training and data collectors' per diem during data collection.

\section{References}

[1] D. Flanagan, T. Fisher, M. Murray et al., "Managing undernutrition in the elderly-prevention is better than cure," Australian Family Physician, vol. 41, no. 9, p. 695, 2012.

[2] UNICEF Tracking Progress on Child and Maternal Nutrition: A Survival and Development Priority, Division of Communication, UNICEF, New York, NY, USA, 2009.

[3] T. D. Hundera, H. F. Gemede, D. Wirtu, and D. N. Kenie, "Nutritional status and associated factors among lactating mothers in nekemte referral hospital and health centers, Ethiopia," International Journal of Nutrition and Food Sciences, vol. 4, no. 2, pp. 216-222, 2015.

[4] F. H. Bitew and D. S. Telake, "Undernutrition among women in Ethiopia: rural-urban disparity," DHS Working Papers No. 77, Technical Report, ICF Macro, Calverton, MD, USA, 2010.
[5] Z. A. Bhutta, J. K. Das, A. Rizvi et al., "Evidence-based interventions for improvement of maternal and child nutrition: what can be done and at what cost?" The Lancet, vol. 382, no. 9890, pp. 452-477, 2013.

[6] M. Alemayehu, A. Argaw, and A. G. Mariam, "Factors associated with malnutrition among lactating women in subsistence farming households from Dedo and Seqa-chekorsa districts, Jimma Zone, 2014," Developing Country Studies, vol. 5, no. 21, pp. 117-118, 2015.

[7] A. Mtumwa, E. Paul, and S. Vuai, "Determinants of undernutrition among women of reproductive age in Tanzania Mainland," South African Journal of Clinical Nutrition, vol. 29, no. 2, pp. 75-81, 2016.

[8] Y. M. Khan and A. Khan, "A study on factors influencing the nutritional status of lactating women in Jammu, Kashmir and Ladakh regions," International Journal of Advancements in Research \& Technology, vol. 1, no. 4, pp. 65-74, 2012.

[9] K. T. Roba, T. P. O'Connor, T. Belachew, and N. M. O’Brien, "Seasonal variation in nutritional status and anemia among lactating mothers in two agro-ecological zones of rural Ethiopia: a longitudinal study," Nutrition, vol. 31, no. 10, pp. 1213-1218, 2015.

[10] "EDHS Ethiopia demographic and health survey 2016, ," Final Report, pp. 1471-2458, CSA and ICF, Addis Ababa, Ethiopia, and Rockville, MD, USA, 2016.

[11] K. Biru, A. Jima, and S. Abeya, "Prevalence of chronic energy malnutrition and maternal health service utilizations among lactating mothers in Adama district, Oromia region, Eastern Ethiopia," Journal of Food Processing and Technology, vol. 8, no. 1, 2017.

[12] “Dega Damot Woreda Health Office Report," 2010.

[13] S. Berihun, G. M. Kassa, and M. Teshome, "Factors associated with underweight among lactating women in Womberma Woreda, Northwest Ethiopia; a cross-sectional study," BMC Nutrition, vol. 3, no. 1, p. 46, 2017.

[14] N. B. Weldehaweria, K. H. Misgina, M. G. Weldu et al., "Dietary diversity and related factors among lactating women visiting public health facilities in Aksum town, Tigray, Northern Ethiopia," BMC Nutrition, vol. 2, no. 1, p. 38, 2016.

[15] FAO and FHI 360 Minimum Dietary Diversity for Women: A Guide for Measurement, FAO, Rome, 2016.

[16] "WHO appropriate body-mass index for asian populations and its implications for policy and intervention strategies," Lancet, vol. 363, no. 9403, pp. 157-163, 2004.

[17] F. Bitew and D. Telake, "Undernutrition among women in Ethiopia: rural-urban disparity," DHS Working Papers No. 77, 2010.

[18] U. P. Ogechi, "A study of the nutritional status and dietary intake of lactating women in Umuahia, Nigeria," American Journal of Health Research, vol. 2, no. 1, pp. 20-26, 2014.

[19] R. E. Black, C. G. Victora, S. P. Walker et al., "Maternal and child undernutrition and overweight in low-income and middle-income countries," The Lancet, vol. 382, no. 9890, pp. $427-451,2013$.

[20] I. K. Sitotaw, K. Hailesslasie, and Y. Adama, "Comparison of nutritional status and associated factors of lactating women between lowland and highland communities of district Raya, Alamata, Southern Tigiray, Ethiopia," BMC Nutrition, vol. 3, no. 1, p. 61, 2017.

[21] K. Haileslassie, A. Mulugeta, and M. Girma, "Feeding practices, nutritional status and associated factors of lactating women in Samre Woreda, south eastern zone of Tigray, Ethiopia," Nutrition Journal, vol. 12, no. 1, p. 28, 2013. 
[22] K. M. Rao, N. Balakrishna, N. Arlappa, A. Laxmaiah, and G. N. V. Brahmam, "Diet and nutritional status of women in India," Journal of Human Ecology, vol. 29, no. 3, pp. 165-170, 2010.

[23] M. Savy, Y. Martin-Prével, P. Traissac, S. Eymard-Duvernay, and F. Delpeuch, "Dietary diversity scores and nutritional status of women change during the seasonal food shortage in rural Burkina Faso," The Journal of Nutrition, vol. 136, no. 10, pp. 2625-2632, 2006.

[24] Z. Wang, S. Dang, and H. Yan, "Nutrient intakes of rural tibetan mothers: a cross-sectional survey," BMC Public Health, vol. 10, no. 1, p. 801, 2010.

[25] D. R. Singh, S. Ghimire, S. R. Upadhayay, S. Singh, and U. Ghimire, "Food insecurity and dietary diversity among lactating mothers in the urban municipality in the mountains of Nepal," PloS One, vol. 15, no. 1, Article ID e0227873, 2020.

[26] D. M. Matheson, J. Varady, A. Varady, and J. D. Killen, "Household food security and nutritional status of hispanic children in the fifth grade," The American Journal of Clinical Nutrition, vol. 76, no. 1, pp. 210-217, 2002.

[27] M. Saaka, "How is household food insecurity and maternal nutritional status associated in a resource-poor setting in Ghana," Agriculture \& Food Security, vol. 5, no. 1, 2016.

[28] W. Girma and T. Genebo, "Determinants of nutritional status of women and children in Ethiopia," DHS Further Analysis Reports No. 39, ORC Macro, Calverton, MD, USA, 2002.

[29] K. L. Hanson, J. Sobal, and E. A. Frongillo, "Gender and marital status clarify associations between food insecurity and body weight," The Journal of Nutrition, vol. 137, no. 6, pp. 1460-1465, 2007.

[30] J. C. King, S. H. Cohenour, D. H. Calloway, and H. N. Jacobson, "Assessment of nutritional status of teenage pregnant girls. I. nutrient intake and pregnancy," The American Journal of Clinical Nutrition, vol. 25, no. 9, pp. 916-925, 1972.

[31] B. Jikamo and M. Samuel, "Does dietary diversity predict the nutritional status of adolescents in Jimma zone, Southwest Ethiopia?” BMC Research Notes, vol. 12, no. 1, p. 402, 2019.

[32] UNICEF, Multi-Sectoral Approaches to Nutrition: NutritionSpecific and Nutrition-Sensitive Interventions to Accelerate Progress, UNICEF, New York, NY, USA, 2016.

[33] S. Abraham, G. Miruts, and A. Shumye, "Magnitude of chronic energy deficiency and its associated factors among women of reproductive age in the kunama population, Tigray, Ethiopia, in 2014," BMC Nutrition, vol. 1, no. 1, p. 12, 2015. 